\title{
Lepton asymmetry effect on neutrino oscillations and primordial ${ }^{4} H e$.
}

\author{
Daniela Kirilova* and Mihail Chizhov $^{\dagger}$ \\ *Institute of Astronomy, Sofia, Bulgaria; e-mail: dani@libra.astro.bas.bg \\ $\dagger$ Theory Division, CERN, Geneva, Switzerland and Sofia University, Sofia, Bulgaria
}

\begin{abstract}
We analyze the effects of lepton asymmetry on neutrino oscillations and on cosmological nucleosynthesis with active-sterile oscillating neutrinos. It is shown that small lepton asymmetries, $L<0.01$, whose direct kinetic effect on nucleosynthesis is negligible, still effect nucleosynthesis considerably through their influence on oscillating neutrinos. Two different cases of lepton asymmetry are discussed: an initially present and a dynamically generated in oscillations. Dynamically generated in resonant oscillations asymmetry at small mixing angles suppresses oscillations, hence, the nucleosynthesis bounds on neutrino mass differences at small mixings are relaxed. Initially present asymmetry may suppress or enhance oscillations. The enhancement is a result of interchanging resonances between neutrino and antineutrino ensembles due to resonance waves passing through the neutrino and antineutrino spectrum. Updated nucleosynthesis bounds on neutrino oscillation parameters accounting for lepton asymmetry are presented.
\end{abstract}

\section{INTRODUCTION}

The direct kinetic effect of a considerable lepton asymmetry $L>0.01$ (either initially present or dynamically generated) on cosmological nucleosynthesis $(\mathrm{CN})$ has been investigated in [1], and cosmological constraints on its value have been obtained. The effect of neutrino oscillations on $\mathrm{CN}$ was also studied and stringent bounds on oscillation parameters were obtained [2-7]. Neutrino oscillations, proceeding in the primordial plasma during $\mathrm{CN}$ epoch, can effect ${ }^{4} \mathrm{He}$ yield by (a) bringing additional degrees of freedom into the primordial heat bath [2], (b) depleting the neutrino and antineutrino number densities ${ }^{1}$ thus slowing the weak interactions $\Gamma_{w} \sim N_{\nu} E_{\nu}^{2}$, (c) distorting the neutrino and antineutrino spectrum [3,4], (d) producing neutrino-antineutrino asymmetry ${ }^{2}[4,8]$, which on its turn influences

1) The effect was estimated for equilibrium [2] and nonequilibrium [3] oscillations and numerically calculated for nonequilibrium oscillations in [4].

2) The asymmetry growth was estimated to be possible for great mass differences $\delta m^{2}>10^{-5}$ $\mathrm{eV}^{2}[8]$, however for $\delta m^{2}<10^{-7} \mathrm{eV}^{2}$, a considerable asymmetry growth was registered in precise numerical studies [4]. 
the evolution of the neutrino and antineutrino ensembles and the oscillation pattern $[4,9,6]$.

In this work we discuss the simultaneous effect of a lepton asymmetry and neutrino oscillations on CN. Two different cases of lepton asymmetry are analyzed: an initially present and generated in oscillations asymmetry. We have studied the role of a lepton asymmetry on $\mathrm{CN}$ with oscillations, effective after electron neutrino decoupling. We have shown by a numerical analyses of the kinetics of nucleons and the oscillating neutrinos and antineutrinos in $\mathrm{CN}$ epoch, that much smaller asymmetries $L<<0.01$ exert considerable indirect effects on $\mathrm{CN}$ through oscillations, due to the fact that even very small asymmetries change the medium induced neutrino potential energy and influence the evolution of the oscillating neutrinos. The prejudice that in order to influence nucleosynthesis the neutrino asymmetry first must grow to a considerable value $L>0.01$, is not applicable for the case of nucleosynthesis with oscillations. ${ }^{3}$

Lepton asymmetry influences CN with oscillations in several ways: The neutrino and antineutrino ensembles evolve differently in comparison with the case without asymmetry, i.e neutrino number densities, their depletion and spectrum distortion are changed. Also due to asymmetry term neutrino and antineutrino become strongly coupled and evolve differently. Lepton asymmetry changes as well the oscillation pattern, i.e. leads to an enhancement or suppression of oscillations. All these resultt into changed light elements yields, compared with the oscillatory case without asymmetry. This indirect asymmetry influence on $\mathrm{CN}$ is considerable [4,5,9-11]. However, inorder to reveal it asymmetry should be considered selfconsistently with the neutrinos and nucleons evolution. The results obtained without the account of the indirect effects differ by many orders of magnitude from the real picture [9]. ${ }^{4}$

Numerical analysis, accounting for the asymmetry effect selfconsistently with neutrino and nucleons evolution, was provided in refs. [5,9,6,7]. See also ref. [14], where an precise analytical study of the asymmetry evolution, accounting for its back effect on oscillating neutrinos, was proposed. In the nonresonant case the oscillations produced asymmetry was shown to have a negligible role in CN [5]. However, in the resonant oscillation case the asymmetry effect on $\mathrm{CN}$ is considerable $[4,9,6,14,7]$.

In general, dynamically produced asymmetry suppresses oscillations [4,9], which leads to less overproduction of helium-4 in comparison with $\mathrm{CN}$ with oscillations but without an asymmetry account. Hence, the bounds on oscillation parameters

3) Really in the case without oscillations the asymmetry has a direct sign-dependent effect on the kinetics of the nucleons, and hence, on the helium yields. Besides, when large enough it also contributes to the Universe energy density thus increasing the expansion rate $H(t)$ and changing primordial helium-4 yield $Y_{p}$ [1].

4) Works considering asymmetry effect on $\mathrm{CN}$ in case of oscillations [12,13], providing rough estimates of the asymmetry growth and its effect on $\mathrm{CN}$, will become more reliable when a proper account for the indirect asymmetry effects on $\mathrm{CN}$ during the full evolution of the asymmetry is provided in a selfconsistent analysis of the neutrino and nucleons evolution for each momentum. 
are alleviated at small mixing angles $[9,6,7]$. We present in sec. III the updated cosmological constraints for electron-sterile oscillation case, accounting precisely for the oscillations generated asymmetry.

The effect of small initial lepton asymmetries $\left(10^{-10}<L<10^{-4}\right)$ on $\mathrm{CN}$ with nonresonant active-sterile oscillations was precisely studied in $[5,10,11]$. It was found that asymmetry is able to enhance oscillations, besides its well known ability to suppress them [10], thus leading correspondingly to an over- or under-production of helium. Our analysis has shown (see sec. IV.) that the initially present asymmetry is able to alleviate $\mathrm{CN}$ bounds at large mixings and to tighten the bounds at small mixings (see also refs. $[10,11]$ ).

In the next section we present the precise kinetic approach for the description of lepton asymmetry effects on $\mathrm{CN}$ in the presence of oscillations.

\section{THE KINETICS}

We have used for the precise analysis the synthesis of helium-4. According to the standard $\mathrm{CN}$ the primordial helium yield depends on two compelling processes, determinning the nucleons freezing: Universe's cooling, $H(t) \sim g_{\text {eff }} T^{2}$ and weak processes, $\Gamma_{w}$. Three neutrino flavours, zero lepton asymmetry and equilibrium neutrino number densities and spectrum are assumed. In the case of CN with oscillations and with lepton asymmetry, all these assumptions do not work. According to (a)-(d): neutrino oscillations change the number of neutrino flavours; they may produce nonequilibrium neutrino number densities (particularly the electron neutrino density may be considerably reduced in favour of the sterile neutrino density); the neutrino spectrum may be distorted in active-sterile oscillations; besides, even if initially the lepton asymmetry is assumed zero resonant oscillations may lead to a considerable growth of the asymmetry, which on its turn effects neutrinos and nucleons evolution via oscillations. This nonequilibrium picture is hard to describe analytically. ${ }^{5}$

Inorder to account for the different effects of oscillations and asymmetry on CN a selfconsistent numerical analysis of the kinetics of the oscillating neutrinos, the

5) There exist in literature numerous analytical studies, like refs. $[12,13]$ proposing different schemes for applying neutrino oscillations to solve different astrophysical or else problems in which schemes a central role is played by a lepton asymmetry (initially present or dynamically generated in oscillations) suppressing oscillations. We would like to warn that these publications provided too rough estimations both of the asymmetry evolution and of its effect on primordial nucleosynthesis. The asymmetry evolution was usually semianalitically described using different sorts of simplifying assumptions, in general not applicable in the nonequilibrium situation of active-sterile oscillations. The neutrino and antineutrino evolution was described in terms of particle densities (not particle density matrix) and almost equilibrium spectrum (which is not an adequate description of oscillationary phenomena when the growth of asymmetry is considerable). The asymmetry effect on nucleosynthesis was reduced only to $L$ kinetic effect on CN. Therefore, its role in nucleosynthesis was accounted for after it has grown "enough" and hence, the indirect effect of asymmetry during its growth has been neglected. The asymmetry back effect on oscillations, in case considered at all, was assumed to be only towards suppressing oscillations. 
nucleons freeze-out and the asymmetry evolution is necessary. In our analysis the set of the following coupled integro-differential equations describing the evolution of the neutrino $\rho$ and neutron number densities $n_{n}$ was solved simultaneously and selfconsistently:

$$
\begin{aligned}
& \frac{\partial \rho(t)}{\partial t}=H p_{\nu} \frac{\partial \rho(t)}{\partial p_{\nu}}+ \\
& \quad+i\left[\mathcal{H}_{o}, \rho(t)\right]+i \sqrt{2} G_{F}\left(\mathcal{L}-Q / M_{W}^{2}\right) N_{\gamma}[\alpha, \rho(t)]+\mathrm{O}\left(G_{F}^{2}\right) \\
& \begin{aligned}
\frac{\partial \bar{\rho}(t)}{\partial t} & =H p_{\nu} \frac{\partial \bar{\rho}(t)}{\partial p_{\nu}}+ \\
& +i\left[\mathcal{H}_{o}, \bar{\rho}(t)\right]+i \sqrt{2} G_{F}\left(-\mathcal{L}-Q / M_{W}^{2}\right) N_{\gamma}[\alpha, \bar{\rho}(t)]+\mathrm{O}\left(G_{F}^{2}\right)
\end{aligned} \\
& \begin{array}{l}
\left(\partial n_{n} / \partial t\right)=H p_{n}\left(\partial n_{n} / \partial p_{n}\right)+ \\
+\int \mathrm{d} \Omega\left(e^{-}, p, \nu\right)\left|\mathcal{A}\left(e^{-} p \rightarrow \nu n\right)\right|^{2}\left[n_{e^{-}} n_{p}\left(1-\rho_{L L}\right)-n_{n} \rho_{L L}\left(1-n_{e^{-}}\right)\right] \\
-\int \mathrm{d} \Omega\left(e^{+}, p, \tilde{\nu}\right)\left|\mathcal{A}\left(e^{+} n \rightarrow p \tilde{\nu}\right)\right|^{2}\left[n_{e^{+}} n_{n}\left(1-\bar{\rho}_{L L}\right)-n_{p} \bar{\rho}_{L L}\left(1-n_{e^{+}}\right)\right]
\end{array}
\end{aligned}
$$

where $\alpha_{i j}=U_{i e}^{*} U_{j e}$, mixing just in the electron sector was assumed $\nu_{i}=U_{i l} \nu_{l}(l=$ $e, s) . p_{\nu}$ is the momentum of electron neutrino, $n$ stands for the number density of the interacting particles, $\mathrm{d} \Omega(i, j, k)$ is a phase space factor and $\mathcal{A}$ is the amplitude of the corresponding process.

These equations provide simultaneous account of the different competing processes, namely: neutrino oscillations, Hubble expansion and weak interaction processes. $\mathcal{H}_{o}$ is the free neutrino Hamiltonian. The 'nonlocal' term $Q$ arises as an $W / Z$ propagator effect, $Q \sim E_{\nu} T . \quad \mathcal{L}$ is proportional to the fermion asymmetry of the plasma and is essentially expressed through the neutrino asymmetries $\mathcal{L} \sim 2 L_{\nu_{e}}+L_{\nu_{\mu}}+L_{\nu_{\tau}}$, where $L_{\mu, \tau} \sim\left(N_{\mu, \tau}-N_{\bar{\mu}, \bar{\tau}}\right) / N_{\gamma}$ and $L_{\nu_{e}} \sim \int \mathrm{d}^{3} p\left(\rho_{L L}-\bar{\rho}_{L L}\right) / N_{\gamma}$.

The neutron and proton number densities, used in the kinetic equations for neutrinos, are substituted from the numerical calculations of eq. (2). On the other hand, $\rho_{L L}$ and $\bar{\rho}_{L L}$ at each integration step of eq. (2) are taken from the simultaneously performed integration of the set of equations (1).

The equations are for the neutrino and neutron number densities in momentum space. This allows to account precisely for the spectrum distortion effect and neutrino depletion effects of oscillations, as well as to follow the evolution of the neutrino asymmetry and its back effect at each neutrino momentum.

In our numerical analysis the spectrum distortion was described by 1000 bins for the nonresonant case and by 5000 bins for the resonant case. In case the spectrum was described by $N$ bins, a system of $6 N+1$ coupled integro-differential equations following from (1) and (2), was numerically solved.

The numerical analysis was provided for the characteristic temperature interval $[2 \mathrm{MeV}, 0.3 \mathrm{MeV}]$ and the full set of oscillation parameters of the active-sterile 


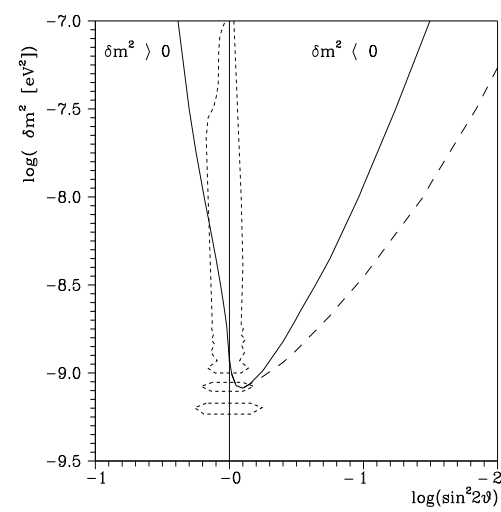

Figure 1. Cosmological constraints for the electron-sterile neutrino oscillations, are presented by the solid curves $Y_{p}=0.24$. The dashed curve shows the contour without asymmetry account. The dotted curve shows solar neutrino LOW solution.

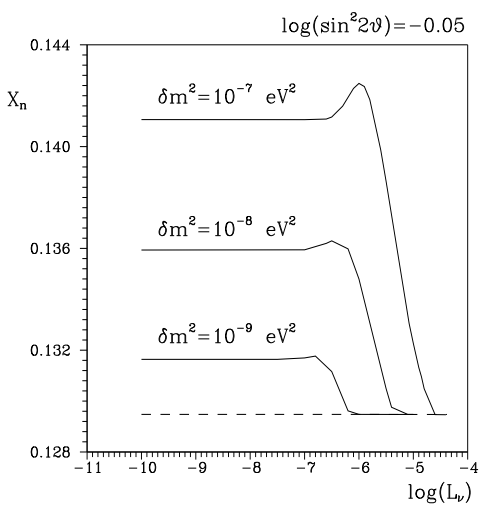

Figure 2. The dependence of the neutron number density relative to nucleons $X_{n}=N_{n} /\left(N_{p}+\right.$ $N_{n}$ ) for the case of oscillations with $\sin ^{2}(2 \vartheta)=10^{-0.05}$ on the value of the initial asymmetry is plotted [10]

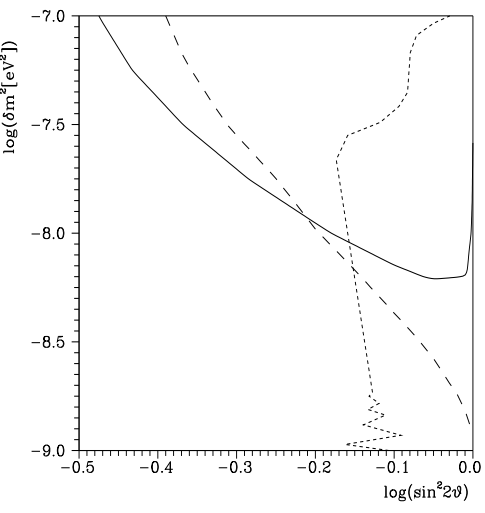

Figure 3. On the $\delta m^{2}-\vartheta$ plane the isohelium contours $Y_{p}=$ 0.24 calculated in the discussed model of CN with neutrino oscillations and initial lepton asymmetries $L=10^{-6}$ (solid curve) and $L=10^{-10}$ are shown. The dot-

oscillation model [4]. We calculated precisely the $n / p$-freezing, essential for the production of helium, till temperature $0.3 \mathrm{MeV}$, and accounted adiabatically for the following decays of neutrons till the start of nuclear reactions at about $0.1 \mathrm{MeV}$.

\section{DYNAMICALLY GENERATED LEPTON ASYMMETRY}

Our numerical analysis showed that in the resonant oscillation case the dynamical neutrino-antineutrino asymmetry grows up to 4 orders of magnitude. I.e. starting with asymmetries of the order of the baryon one it reaches maximum a value $10^{-5}$. Hence, having in mind this small value the registered asymmetry effect is totally due to its indirect influence on $\mathrm{CN}$ via oscillations. Dynamically produced asymmetry at small mixing angles suppresses oscillations, which leads to less overproduction of ${ }^{4} \mathrm{He}$ in comparison with $\mathrm{CN}$ with oscillations but without an asymmetry account. Hence, the cosmological constraints on oscillation parameters are alleviated at small mixing angles.

The updated constraints on active-sterile neutrino oscillations, precisely accounting for the asymmetry generation, spectrum distortion and the depletion of the neutrinos, are presented in fig. 1. The plots correspond to $Y_{p}=0.24$. The net indirect asymmetry effect on $\mathrm{CN}$ is given for the resonant case by the difference between the dashed curve (without asymmetry account) and the solid one. Due to asymmetry growth account, and the corresponding suppression of oscillations, $Y_{p}$ overproduction is not so strongly expressed at small mixing angles, hence $\mathrm{CN}$ constraints are alleviated at small mixing angles for $\delta m^{2}<0$.

In the resonant case the cosmological constraints at large mixings are $\left|\delta m^{2}\right| \leq$ $8.2 \times 10^{-10} \mathrm{eV}^{2}$. In the nonresonant case $\delta m^{2}>0$ an analytical fit to the exact constraints is: $\delta m^{2}\left(\sin ^{2} 2 \vartheta\right)^{4} \leq 1.5 \times 10^{-9} \mathrm{eV}^{2}$. The constraints in both cases are 
strengthened compared to the previous ones [2] due to the precise account of the spectrum distortion and to the exact kinetic approach to the neutrinos and nucleons evolution.

According to these constraints the LOW active-sterile solution to the solar neutrino problem, which is favoured by the analysis of the recent experimental data of total measured rates and day and night spectrum measured by $\mathrm{Su}-$ perKamiokande [15] is almost completely excluded [11].

\section{INITIALLY PRESENT LEPTON ASYMMETRY}

The role of initially present relic asymmetries on $\mathrm{CN}$ with nonresonant activesterile oscillations was precisely studied, following the lines of work described in sec. II. A wide range of $L$ values $\left[10^{-10}-10^{-4}\right]$ was analyzed. Such small asymmetries have only indirect effects on CN. On fig.2 the dependence of the produced helium, in a CN model with oscillations on the initial asymmetry is plotted. It was found that the asymmetry is able also to enhance oscillations, besides its well known ability to suppress them. The enhancement is a synthetic effect of a resonant wave passing through neutrino spectrum till lepton asymmetry changes sign and followed by a similar 'spectrum' resonance at the antineutrino ensemble and vice versa [10]. Hence, this enhancement has a complex spectral character and could be revealed only by a precise kinetic approach to the oscillation problem. The influence of the asymmetry should not be reduced only to oscillations suppression, as usually believed. Depending on the concrete values of oscillations parameters the asymmetry may suppress, enhance or not influence oscillations, thus leading correspondingly to an under-, over-production of helium or not change its abundance. Hence, in order to judge the real asymmetry effect on $\mathrm{CN}$, precise numerical analysis for the concrete oscillation parameters and $L$ values is obligatory. Initially present asymmetry may relax $\mathrm{CN}$ bounds at large mixings and tighten the bounds at small mixings.

Qualitatively, for oscillations affective after the freeze-out of the electron neutrino, the asymmetry effect is as follows: $L<10^{-7}$ has negligible effect on $\mathrm{CN}$; $10^{-7}<L<10^{-5}$ enhances oscillations due to the spectrum wave resonance [10], resulting into an enhanced overproduction of helium-4; $L>10^{-5}$ leads to a suppression of oscillations and relaxation of the $\mathrm{CN}$ bounds (fig. 2).

In fig. 3 the isohelium contours $Y_{p}=0.24$ are presented, for different $L: L=$ $10^{-6}$ and $L=10^{-10}$. For small mixing angles the asymmetry $L=10^{-6}$ enhances oscillations, which reflects into stronger bounds on oscillation parameters, while for large mixings this asymmetry suppresses oscillations and $\mathrm{CN}$ bounds are weakened compared with $L=10^{-10}$ case.

A similar investigation, of initial asymmetry effect on $\mathrm{CN}$ with oscillations, for the resonant case will be a more complicated task due to technical problems: The usual explicit numerical approach is not applicable for the description of the asymmetry evolution, because the neutrino evolution equations at resonance have high 
stiffness; besides the resonance case deserve much greater number of bins for the spectrum distortion description. To solve the stiff equations numerically, implicit methods should be used. For 5000 bins of the spectrum a system of 30000 equations describing the neutrino evolution should be solved simultaneously. However, this investigation is interesting, as far as such small values of the initial relic asymmetry are not excluded neither from observations nor from some profound theoretical principle.

In conclusion we would like to stress that small asymmetries, initially present or dynamically generated, influence CN thanks to their backfeed effect on oscillating neutrinos. The account of spectrum distortion of the oscillating neutrinos as well as the selfconsistent account of neutrinos and nucleons evolution is essential for revealing the indirect effect of small lepton asymmetries. Therefore, a precise kinetic approach should be provided when analyzing the effect of lepton asymmetry on CN with oscillations.

We are glad to thank the organizing committee of CAPP2000, for the stimulating

atmosphere of the conference and for the financial support of the participation of D.K. M.C. thanks Theory Division of CERN where this work was prepared.

\section{REFERENCES}

1. Wagoner R. V., Fowler W. A., and Hoyle F., Astrophys. J. 148, 3 (1967); Terasawa N., and Sato K., Prog. Theor. Phys. 80, 468 (1988) and the references there in.

2. Dolgov A. D., Sov. J. Nucl. Phys. 33, 700 (1981); Barbieri R., and Dolgov A., Phys. Lett. B 237, 440 (1990); Nucl. Phys. B 349, 743 (1991); Enqvist K., Kainulainen K., and Thomson M., Nucl. Phys. B 373, 498 (1992); Dolgov A. D., hep-ph/0006103.

3. Kirilova D. P., JINR preprint E2-88-301, 1988.

4. Kirilova D. P., and Chizhov M. V., in Proc. NEUTRINO 96 Conference, Helsinki, 1996, p. 478; Phys. Lett. B 393, 375 (1997)

5. Kirilova D. P., and Chizhov M. V., Phys. Rev. D 58, 073004 (1998).

6. Kirilova D. P., and Chizhov M. V., Nucl. Phys. B 591, 457 (2000).

7. Kirilova D., talk at Astrophysics Workshop "Hot Points in Astrophysics", August 2000, Dubna, Russia, to be published in the proceedings.

8. Foot R., Thomson M. J., and Volkas R. R., Phys. Rev. D 53, R5349 (1996); Shi X., Phys. Rev. D 54, 2753 (1996).

9. Kirilova D. P., and Chizhov M. V., hep-ph/9908525.

10. Kirilova D. P., and Chizhov M. V., Nucl. Phys. B 534, 447 (1998).

11. Kirilova D., talk at NOW2000 Workshop, 11-16 Sept. 2000, Otranto, Italy, to be published in Nucl. Phys.B.

12. Abazajian K., Shi X., and Fuller G. M., astro-ph/9904052; Shi X., Fuller G. M., and Abazajian K., astro-ph/9908081; Phys. Rev. D 60, 063002 (1999); Shi X., and Fuller G. M., Phys. Rev. Lett. 83, 3120 (1999).

13. Foot R., and Volkas R. R., Phys. Rev. D 55, 5147 (1997); ibid. D 56, 6653 (1997); Erratum ibid. D 59, 029901 (1999); Phys. Rev. D 61, 043507 (2000); astro-ph/9811067; 
Bell N., Foot R., and Volkas R. R., Phys. Rev. D 58, 105010 (1998); Foot R., Astropart. Phys. 10, 253 (1999); Phys. Rev. D 61, 023516 (2000); Di Bari P., Lipari P., and Lusignoli M., Int. J. Mod. Phys. A 15, 2289 (2000).

14. Dolgov A. et al. hep-ph/9910444, TAC-1999-018.

15. Suzuki Y., talk at Neutrino 2000, June 2000, Sudbury, Canada; Gonzalez-Garcia M. C., Peña-Garay C., hep-ph/0009041 talk at Neutrino 2000, June 2000, Sudbury, Canada; Bahcall J. N., Krastev P. I., and Smirnov A. Yu., talk at NOW2000, Sept. 2000, Otranto, Italy. 